\title{
Correction to: Endometrium metabolomic profiling reveals potential biomarkers for diagnosis of endometriosis at minimal-mild stages
}

\author{
Jingjie $\mathrm{Li}^{1+}$, Lihuan Guan ${ }^{2 \dagger}$, Huizhen Zhang ${ }^{2}$, Yue Gao ${ }^{2}$, Jiahong Sun ${ }^{2}$, Xiao Gong ${ }^{4}$, Dongshun Li ${ }^{2}$, Pan Chen ${ }^{3}$, \\ Xiaoyan Liang ${ }^{1}$, Min Huang ${ }^{2}$ and Huichang $\mathrm{Bi}^{2^{*}}$
}

\section{Correction to: Reprod Biol Endocrinol (2018) 16:42 https://doi.org/10.1186/s12958-018-0360-z}

The authors regret that the incorrect identification of "omega-3 arachidonic acid" published in the original manuscript [1]. This classification is incorrect as arachidonic acid is an omega- 6 polyunsaturated fatty acid. From a mechanistic standpoint, this mistake might have significance, as arachidonic acid is hypothesized to play a role in inflammatory processes. We have carefully double-checked our original data and compared the fragmentation spectra and $\mathrm{m} / \mathrm{z}$ referring to Metlin Database again. We are sure that the metabolite should be arachidonic acid, instead of omega-3 arachidonic acid. Given that this misclassification is made in several places within the article's abstract and text, a correction is warranted.

Accordingly, the name "omega-3 arachidonic acid" mentioned in the following parts should be corrected as "arachidonic acid":

- In the results section of Abstract: "The eutopic endometrium metabolomic profile of the endometriosis patients was characterized by a significant increase in the concentration of hypoxanthine, L-arginine, L-tyrosine, leucine, lysine, inosine, omega-3 arachidonic acid, guanosine.......".

\footnotetext{
* Correspondence: bihchang@mail.sysu.edu.cn

The original article can be found online at https://doi.org/10.1186/s12958 018-0360-z

${ }^{\dagger}$ Jingjie Li and Lihuan Guan contributed equally to this work.

${ }^{2}$ School of Pharmaceutical Sciences in Sun Yat-sen University, 132\#

Waihuandong Road, Guangzhou, University City, Guangzhou 510006,

People's Republic of China

Full list of author information is available at the end of the article
}

- In the last paragraph of Introduction: "The eutopic endometrium metabolomic profile of endometriosis patients was characterized by a significant increase in the concentration of hypoxanthine, L-arginine, L-tyrosine, leucine, lysine, inosine, omega-3 arachidonic acid, guanosine......".

- In the first paragraph of the section with the subheading "Identification of detected metabolites" of Results: "Obviously, levels of hypoxanthine, Larginine, L-tyrosine, leucine, lysine, inosine, omega-3 arachidonic acid, guanosine....."

- In the first paragraph of Discussion: "In this regard, 11 metabolites including hypoxanthine, L-arginine, L-tyrosine, leucine, lysine, inosine, omega-3 arachidonic acid, guanosine......"

- Figure 2D, "Omega-3 Arachidonic acid"

- In the third row of Figure 3, "omega-3 arachidonic acid"

- In the third graph in the first row of Figure 4, "Omega-3 Arachidonic acid"

- In the 4th row of RPLC mode in Table 2, "Omega-3 Arachidonic acid"

The authors would like to apologize for any inconvenience caused. 


\section{Author details}

${ }^{1}$ Center of Reproductive Medicine, the Sixth Affiliated Hospital, Sun Yat-sen

University, Guangzhou, China. ${ }^{2}$ School of Pharmaceutical Sciences in Sun Yat-sen University, 132\# Waihuandong Road, Guangzhou, University City,

Guangzhou 510006, People's Republic of China. ${ }^{3}$ Pharmacy Department, the First Affiliated Hospital, Sun Yat-sen University, Guangzhou, China. ${ }^{4}$ School of Public Health, Guangdong Pharmaceutical University, Guangzhou, China.

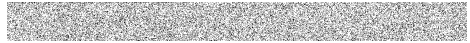

\section{Reference}

1. Li J, et al. Endometrium metabolomic profiling reveals potential biomarkers for diagnosis of endometriosis at minimal-mild stages. Reprod Biol Endocrinol. 2018;16:42. https://doi.org/10.1186/s12958-018-0360-z. 